We appreciate the response to this publication feature and welcome all contributions. Contributions may be sent to José A. Mascorro, our Technical Editor, at his e-mail address: jmascor@tulane.edu. José may also be reached at the Department of Structural and Cellular Biology, Tulane University Health Sciences Center, 1430 Tulane Ave., New Orleans, LA 70112 and $\mathrm{Ph}:(504) 584-2747$ (504) 584-1687

\section{Comments on Cryo High Resolution Scanning Electron Microscopy}

\author{
Robert P. Apkarian \\ IM\&MF/Emory University \\ rapkari@emory.edu
}

Stephen Carmichael wrote about Cryoelectron Tomography in the May 2003 issue of Microscopy Today. Citing new preparation methods, small cells can be vitrified, observed frozen in the TEM and a series of digital images captured while the specimen is being rotated around the axis perpendicular to the electron beam producing a 3-D tomogram. Gina Sosinski and Maryann Martone wrote about imaging big and messy biological structures using cryo-electron Tomography in the July issue of Microscopy Today. Cryo-HRSEM now also seeks to provide 3-D information approaching the molecular level from frozen hydrated cell and molecular systems. Vitrification procedures for small specimens such as platelets and biomolecules on grids are accomplished by plunge freezing in liquefied ethane as is done with cryo-TEM procedures. Bulk specimens such as organic hydrogels and tissues are routinely high pressure frozen (HPF) in $3 \mathrm{~mm}$ gold planchets. Employing an in-lens cryostage, identical to those used in cryo-TEM, cryo-HRSEM provides 3-D high-resolution images because secondary electrons are efficiently collected above the lens in a single scan thus minimizing specimen irradiation. Further cryo HRSEMs employ beam energies of $5-30 \mathrm{kV}$ verses the $100-300 \mathrm{kV}$ used in cryo-TEM Tomography. Certainly the current trends in structural cell studies heavily employ cryo-TEM Tomography. Although cryo-HRSEM has not achieved the popularity of cryo-TEM, several labs have made significant advances in structural analysis.

In the June and August 2003 issues of Microscopy and Microanalysis several articles appear that parallel the "High Resolution Cryo SEM in the Biological Sciences" symposium at M\&M 2003. In the 1990 s several labs using direct cryo-HRSEM imaging showed quality micrographs with structural resolution of a few nanometers on intramembraneous particles representing transmembrane proteins from the protoplasmic fracture face of frozen hydrated yeast cells rather than indirect platinum replicas viewed by TEM.1,2 More recently, P. Walther showed 3-D images of ribosomes and lumenal spaces in the rough endoplasmic reticulum of frozen-sectioned pancreatic tissue, while T. Allen investigated the molecular components of nucleopores from isolated nuclei and within oocyte samples. Apkarian, Wright, and Conticello have published two papers using cryoetch-HRSEM of bioorganic hydrogels including elastin polymers. These lipid and protein hydrogel systems when etched have a striking resemblance to cytoskeletal and membrane-like cell components and reveal filamentous protein structures in the same size range $(\sim 7 \mathrm{~nm})$ as human tropoelastin. As more facilities develop cryo-HRSEM capabilities this method will join the arsenal of molecular cryo-imaging.

\section{References}

1. E. R. Wright et al. Microsc, and Microanal. 2003 9:3 171-182.

2. Microsc, and Microanal. 2003 9:4 272-295.

\section{Propylene Oxide: To Use or Not to Use in Biological Tissue Processing}

\author{
Jose A. Mascorro \\ Tulane University Health Sciences Center \\ jmascor@tulane.edu
}

Propylene oxide (1,2-epoxy propane) has long been utilized as a transitional solvent in the preparation of biological tissues. This com- pound is completely miscible with the various epoxy resins utilized for infiltrating tissues. Ethanol, perhaps the most widely used dehydration agent, does not possess this characteristic and must be removed before the process of infiltration commences. It is common practice amongst biological microscopists to pass tissues for a few minutes through pure propylene oxide $(\mathrm{PO})$ immediately prior to infiltration. The tissues then are pre-infiltrated with various proportions of $\mathrm{PO}$ and the embedding medium of choice. For example, one traditional recipe takes the tissues progressively from pure $\mathrm{PO}$ to $2: 1 \mathrm{PO} /$ resin, $1: 1 \mathrm{PO} /$ resin, and 1:2 $\mathrm{PO} /$ resin mixtures, with stays of a few minutes at each stage. A lengthy procedure such as this probably is not necessary and has never been favored by this worker. Nevertheless, because epoxy resins readily mix with PO, this useful compound is very much in use as it slowly and effectively transitions the tissues from alcohol to the full embedding medium to begin infiltration. It is worth noting that epoxy resins and the full embedding media prepared with them also are miscible, at least to some extent, with ethanol (Table I).

The rationale for utilizing $\mathrm{PO}$ as a transitional solvent is quite sound. In addition to being an excellent reactive diluent, it also will readily combine with anhydride hardeners in the embedding medium. Thus, it becomes an integral part of the cross-linked polymer. Small amounts of $\mathrm{PO}$ remaining unbound apparently will not cause problems with embedding, polymerization, and sectioning. There is evidence, however, that $\mathrm{PO}$ easily extracts lipids, particularly if tissues have not received post-fixation with osmium tetroxide. $\mathrm{PO}$ also will interact with reactive groups in the cells and possibly interfere with histochemical and staining reactions.

Microscopists working with $\mathrm{PO}$ certainly are aware of its health hazards. It is known as an irritant, carcinogenic and flammable organic substance. It can cause skin irritation, contact dermatitis, respiratory distress, and easily penetrates laboratory gloves. This group of real or potential health hazards requires ultimate care when handling this material.

The results presented in Table I indicate clearly that $\mathrm{PO}$ is most effective as a diluent. When mixed in a $1: 1$ ratio with $P O$, the stock resin mixture (A) quickly becomes less viscous $(1.60 \mathrm{cp})$ than when the same mixture is similarly combined with alcohol $(4.17 \mathrm{cp})$. Thus, $\mathrm{PO}$ produces a very fluid combination that would seem more amenable to initial infiltration. On the other hand, even while alcohol is not as effective at reducing viscosity, this does not seem to prove problematical in terms of initial infiltration. Tissues can be passed through a $1: 1$ mixture of alcohol and embedding medium for a 15 minute period, with constant agitation on a rotator. This is followed by full infiltration for $45-60$ minutes, also with agitation. Or infiltration can be accomplished by utilizing whatever specific schedule is favored by the microscopist.

Early training more or less programmed this microscopist to utilize $\mathrm{PO}$ as a matter of routine. However, it has become apparent in recent years that eliminating the entire PO transitional step may be an attractive alternative to the use of $\mathrm{PO}$. This works well when one is processing soft biological (organ) tissues. Not only would this shorten the length of overall tissue processing, but it also would subject the worker to one less, of many, dangerous chemicals.

\begin{tabular}{|c|c|c|c|}
\hline \multicolumn{4}{|c|}{ Table I: Viscosity of LX 112} \\
\hline \multicolumn{4}{|c|}{ Propylene Oxide vs Ethanol } \\
\hline \multicolumn{4}{|c|}{ Stock resin mixture (A): LX112, DDSA, NMA } \\
\hline Substance: & Density: & Temperature: & Viscosity: \\
\hline A & 1.167 & $21.5^{\circ} \mathrm{C}$ & $206 \mathrm{cp}$ \\
\hline $\mathrm{A}+\mathrm{PO}(1: 1)$ & 0.98 & $21.5^{\circ} \mathrm{C}$ & $1.60 \mathrm{cp}$ \\
\hline A+Ethanol & 0.99 & $21.5^{\circ} \mathrm{C}$ & $4.17 \mathrm{cp}$ \\
\hline
\end{tabular}

\section{References}

1. Glauert, A.M. and P.R. Lewis. Practical Methods in Electron Microscopy: Biological Specimen Preparation for Transmission Electron Microscopy, Volume 17. Princeton University Press, Princeton, New Jersey, 1998.

2. Hayat, M.A. Principles and Techniques of Electron Microscopy, 3rd edition. CRC Press, Inc. Boca Raton, Florida, 1989.

3. The author gratefully acknowledges Dr, Peter Schofield from Ladd Research, Inc for providing viscosity information. 